Nouvelles perspectives en sciences sociales

Sociologie de la musique. La construction sociale du son des

" tribus " au numérique, Lello Savonardo, Paris, Éditions

Academia-L'Harmattan, 2015

\title{
Sébastien Dol
}

Volume 13, numéro 2, mai 2018

URI : https://id.erudit.org/iderudit/1051119ar

DOI : https://doi.org/10.7202/1051119ar

Aller au sommaire du numéro

Éditeur(s)

Prise de parole

ISSN

1712-8307 (imprimé)

1918-7475 (numérique)

Découvrir la revue

Citer ce compte rendu

Dol, S. (2018). Compte rendu de [Sociologie de la musique. La construction sociale du son des " tribus » au numérique, Lello Savonardo, Paris, Éditions Academia-L'Harmattan, 2015]. Nouvelles perspectives en sciences sociales, 13(2), 296-299. https://doi.org/10.7202/1051119ar d'utilisation que vous pouvez consulter en ligne.

https://apropos.erudit.org/fr/usagers/politique-dutilisation/ 


\section{Sociologie de la musique. La construction sociale du son des "tribus " au numérique}

Lello Savonardo, Paris, Éditions Academia-L'Harmattan, 2015.

\section{PAR SÉbastien Dol \\ Université Sainte-Anne, \\ Pointe-de-l'Église (Nouvelle-Écosse)}

Traduit de l'italien par Malika Combes et Amélie Munoz

Traduit de l'italien, Sociologie de la musique. La construction présentée sur huit chapitres et divisée en deux sections. Le livre établit un lien entre la théorie de la sociologie classique et la situation actuelle du langage musicale dans une optique sociale et culturelle contemporaine. Pour cette raison, ce livre sert de point de départ pour celui qui désire s'initier à la sociologie de la musique et de matière à réflexion pour le chercheur chevronné.

La préface de l'édition française est signée par Marco Martiniello. Ce dernier indique que la traduction de cette ouvre comble un vide dans le domaine. Dans la préface de l'édition originale, Franco Crespi, parle de façon générale de la sociologie de l'art et de son lien avec la production artistique et musicale.

Dans la première partie de cette étude, qui porte sur la sociologie de la musique, Lello Savonardo examine la contribution théorique des grands penseurs de cette discipline. Son but est de montrer comment elle s'applique au domaine de la musique. Parmi les auteurs présentés, on trouve Georg Simmel, Max Weber, Theodor W. Adorno et Alfred Schütz. Dans la deuxième partie, Savonardo se concentre sur les idées plus contemporaines de Howard S. Becker et de Pierre Bourdieu et jette un coup d'œil sur la manière dont les produits culturels et musicaux sont créés par les sociétés et reçus par elles.

Dans le premier chapitre, "Georges Simmel et les études psychologiques et ethnologiques de la musique ", l'auteur 
explique que, dans ses recherches, Simmel se penche sur les origines de la musique et tente d'examiner non seulement les processus musicaux, mais aussi la relation entre l'artiste et l'auditeur ainsi que les relations qui existent entre l'œuvre, l'individu et la société. Dans ce chapitre, on aborde les thèmes de l'art et la mode ainsi que les origines de la musique. Cet examen se termine en une section de réflexions critiques.

Le livre se poursuit avec «Max Weber et la construction sociale de la tonalité ». Dans ce deuxième chapitre, on peut lire que le point de repère pour Weber, sociologue allemand, c'est l'étude du lien entre les formes musicales et la société selon le processus de rationalisation. Cette étude de l'approche wébérienne instruit le lecteur sur la naissance de la sociologie de la musique et permet de distinguer les travaux de Weber de ceux de Simmel. Le chapitre se termine avec une section critique de la pensée wébérienne.

Le troisième chapitre, intitulé "Theodor W. Adorno, musique et théorie critique ", traite des relations entre les phénomènes musicaux, les consommateurs de la musique, l'idéologie et les classes sociales. Selon Savonardo, Adorno montre que la musique, qui est un produit de la société, sert aussi à critiquer le système social en place. Savonardo touche à ce point surtout sur à la fin du chapitre, dans la section " Jazz et popular music, entre totalitarisme et régression de l'écoute ".

Enfin, pour conclure la première section du livre, l'auteur présente la pensée de Schütz et la phénoménologie de la musique. Schütz approche le sujet de la sociologie de la musique sous l'angle de l'intersubjectivité, nous explique Savonardo. Cela oblige donc à un examen de l'interaction entre le compositeur, l'interprète et le public. De plus, l'approche de Schütz inclut non seulement le partage mais aussi l'interprétation de l'œuvre musicale.

Dans un survol de la seconde partie du livre, Savonardo aborde les concepts de médias et culture de masse en lien avec la musique. Dans le cinquième chapitre ("Mondes artistiques et distinction sociale »), on peut en apprendre plus sur la pensée de Howard S. Becker et de Pierre Bourdieu. C'est ici que Savonardo 
considère les relations entre la société et les formes de production et de réception de l'art. Cette expression et consommation d'art est en changement, nous dit-il, et, au sixième chapitre (" Popular music, médias de masse et culture juvénile »), Savonardo parle de la musique populaire en faisant un survol de ce que c'est, de son évolution et de l'hybridation de la chanson. Le regard sur la transformation dans la musique, ses différentes techniques de création et le rapport qui existe entre la musique et la technologie nous plonge dans le septième chapitre ("Les technologies de la musique »). Dans celui-ci, Savonardo présente l'évolution de la consommation de la musique, en passant par la consommation domestique, la radiodiffusion et même la consommation plus personnalisée et portative avec l'avènement du Walkman. Au chapitre huit ("Musique, nouveaux médias et culture numérique "), il décrit la transformation de l'analogique, avec les disques vinyle et la bande magnétique, au numérique, aux disques compacts (CD) dans les années 1980, aux fichiers mp3 et à l'iPod. Avec la disponibilité de Wi-Fi, le partage des fichiers numériques devient pratique courante et permet un rapprochement dans le partage de fichiers dans ce "village globale " créé par l'internet.

Dans la conclusion, intitulée "La construction sociale de l'expérience sonore ", Savonardo propose aux lecteurs cinq grands thèmes pour une étude sociologique de la musique : Connexions, Innovations, Créativité, Interactivité et Parcours. Il est intéressant de noter que Savonardo ne fait allusion à la première partie de son livre dans sa conclusion que très brièvement et qu'il se concentre sur ces grands thèmes qui tiennent compte de l'impact de la technologie sur la musique, la société, le créateur, le consommateur et des influences que tous ces composants ont les uns sur les autres.

Cet ouvrage est une ressource intéressante, qui présente non seulement les contributions de penseurs de référence pour la sociologie de la musique, mais qui retrace dans un deuxième lieu, l'évolution technologique et la manière dont elle se rattache à la consommation et à la production musicale. Une des mérites de 
ce livre est que l'auteur prend le temps d'énumérer les forces et les faiblesses des travaux des penseurs qu'il a présentés. Il tient compte de multiples points de vue et met tout cela de l'avant, sans prétention. Enfin, l'œuvre est couronnée d'une bibliographie de 30 pages qui comprend au-delà de 330 entrées individuelles. Pour le chercheur en sociologie de la musique, pour celui qui s'intéresse aux médias de masses et à la Popular music, il sera difficile de trouver une liste de ressources aussi complète. 